\title{
Fragmentation-Aware Traffic Grooming with Lane Changes in Spectrally-Spatially Flexible Optical Networks
}

\author{
Piotr Lechowicz *(D), Aleksandra Knapińska (D) and Róża Goścień (D) \\ Department of Systems and Computer Networks, Wrocław University of Science and Technology, \\ Wyspiańskiego 27, 50-370 Wrocław, Poland; Aleksandra.Knapinska@pwr.edu.pl (A.K.); \\ Roza.Goscien@pwr.edu.pl (R.G.) \\ * Correspondence: Piotr.Lechowicz@pwr.edu.pl
}

Citation: Lechowicz, P.; Knapińska,

A.; Goścień, R. Fragmentation-Aware Traffic Grooming with Lane Changes in Spectrally-Spatially Flexible Optical Networks. Electronics 2021, 10, 1502. https://doi.org/10.3390/

electronics10121502

Academic Editor: Martin Reisslein

Received: 20 May 2021

Accepted: 12 June 2021

Published: 21 June 2021

Publisher's Note: MDPI stays neutral with regard to jurisdictional claims in published maps and institutional affiliations.

Copyright: (c) 2021 by the authors. Licensee MDPI, Basel, Switzerland. This article is an open access article distributed under the terms and conditions of the Creative Commons Attribution (CC BY) license (https:// creativecommons.org/licenses/by/ $4.0 /)$.

\begin{abstract}
Traffic in current networks is constantly increasing due to the growing popularity of various network services. The currently deployed backbone optical networks apply wavelength division multiplexing (WDM) techniques in single-core single-mode fibers (SMFs) to transmit the light. However, the capacity of SMFs is limited due to physical constraints, and new technologies are required in the near future. Spectrally-spatially-flexible optical networks (SS-FONs) are proposed to provide a substantial capacity increase by exploring the spatial dimension. However, before this technology will reach maturity, various aspects need to be addressed. In particular, during traffic grooming, multiple small requests are aggregated into large-capacity optical corridors in an optical layer to increase the spectral efficiency. As the summary traffic volume is dynamically changing, it may be required to set up and tear down optical channels, which results in network fragmentation. As a consequence, in a congested network, part of the requests can be blocked due to the lack of spectrum resources. Thus, the grooming of traffic and the creation of lightpaths should be carefully designed to minimize network fragmentation. In this study, we present several fragmentation metrics and develop a fragmentation-aware traffic grooming algorithm that reduces the bandwidth blocking probability.
\end{abstract}

Keywords: modeling and optimization of optical networks; spectrally-spatially flexible optical networks; routing, spectrum, and space allocation; traffic grooming; network fragmentation; lane changes

\section{Introduction}

The continuous growth of traffic in the backbone networks has been observed, aiming to mitigate the constantly increasing requirements of various network services. According to a forecast [1], global IP traffic is increasing approximately with a $26 \%$ compound annual growth rate, mostly affected by the inter-data center or client-to-data center traffic. Most of the currently deployed backbone optical networks apply wavelength-division multiplexing (WDM) techniques over single-core single-mode fibers (SMFs) to deliver the traffic. The nonlinear Shannon limit defines the maximum capacity (information rate) that can be transmitted with the arbitrarily low bit error rate. As the signal in the fiber is prone to noise and interference, the available capacity in SMFs is expected to be exhausted in the near future, and the replacement of optical backbone network is required $[2,3]$. Spectrally-spatially-flexible optical networks (SS-FONs) are seen as a promising solution to overcome the future "capacity crunch". In more detail, SS-FONs combine two independent technologies: spatial division multiplexing (SDM) and elastic optical networks (EONs) $[4,5]$. SDM allows substantially increasing the amount of transmitted traffic by exploiting the spatial dimension. To this end, suitably designed optical fibers are required where a signal is co-propagated in spatial modes. One of the proposed optical fibers is a single-core single-mode fiber bundle (SMFB), which aggregates several SMFs in a bundle [6]. Another drawback of WDM is the rigid spectrum allocation within broad boundaries, resulting 
in underutilization of spectrum resources for small requests and a lack of direct support for larger ones. EONs divide the available spectrum into a flexible grid composed of narrow slots (slices) to support the allocation of heterogeneous requests using just-enough spectrum resources by dynamically adjusting the optical bandwidth and modulation format (MF). In more detail, the optical corridors, called super-channels (SChs), can be created in various sizes, each comprised of one or more adjacent (contiguous) spectrum slots. Routing and spectrum assignment (RSA) is a fundamental problem in EONs where lightpaths are assigned to the requests. The problem is challenging due to the continuity constraint, which implies that in the case of a lack of spectrum converters, each SCh has to occupy slots with the same indices in each link of the routing path $[7,8]$.

A combination of SDM and EONs allows creating SChs in the spectrum and/or spatial domain depending on the fiber and network nodes' architecture, allowing for high allocation flexibility. Analogous to the spectrum continuity constraint in EONs, the spatial continuity constraint (SCC) in SDM can be considered. In the case of the SCC, lane changes in network nodes are not allowed, i.e., having adequately indexed spatial modes in each network link, a request can be transmitted only using a set of spatial modes with the same indices on each link of the routing path. On the contrary, when the SCC is relaxed, each SCh can be transmitted over a different spatial mode of each fiber along the routing path $[4,5]$. Note the fact that whether the SCC is considered is enforced by the lane change ability of the network nodes' architecture. Recently, two network nodes' architectures were proposed, with and without the support of lane changes, namely broadcast and select (B\&S) fully non-blocking (FNB) SDM-capable reconfigurable add and drop multiplexers (ROADMs) and $B \& S$ core continuity constraint (CCC) SDM-capable ROADMs. Lane changes increase the flexibility of request allocation; however, the complexity and cost of network nodes are significantly increased due to the required higher number of spectrum selective switches (SSSs) $[9,10]$.

One of the fundamental problems in the design and operation of SS-FONs is routing, spectrum, and space allocation (RSSA) assignment. Having a set of requests, it refers to finding a routing path and a feasible optical corridor for each of them. An optical corridor is feasible if it is not assigned to any other request and if it satisfies the continuity and contiguity constraints in the spectral domain (and in the spatial domain depending on the network nodes' architecture). Note, the RSSA problem in SS-FONs is at least as challenging as the RSA problem in EONs, due to the additional spatial domain $[6,11,12]$.

The problem becomes even more challenging in a dynamic scenario where requests can have different lifetimes. Thus, the resources should be assigned and released adequately, and the current decisions may affect the performance of the network in the future. In particular, the repeated setup and release of SChs increase network fragmentationthe creation of small gaps in the spectrum domain, which are difficult for the next requests to utilize. As the fragmentation of the network builds up, it becomes harder to find optical corridors for incoming requests, and eventually, they become rejected due to the lack of resources. The proper network management and allocation policy can reduce the blocking probability of requests using proactive and reactive techniques. In proactive techniques, resources for the requests are assigned, aiming to minimize the network fragmentation, while in reactive ones, requests are reallocated either periodically or after reaching a certain threshold of rejections. In particular, various metrics can be designed to quantitatively describe network fragmentation. Properly designed metrics should correlate with the blocking probability; thus, the minimization of that metric in an allocation algorithm can increase the network performance [13]. Note that the topic of network fragmentation has been extensively studied in the context of EONs (see [13-15]); however, it is relatively new for SS-FONs. Introducing the additional spatial dimension causes the metrics and algorithms designed for EONs to be unable to directly be applied in the optimization of SS-FONs, especially when lane changes are considered. As shown in [16], the choice of the fragmentation metric has an influence on the overall bandwidth blocking in the network, as they approach the issue accounting for different network properties. 
To further improve the spectral efficiency of a network, optical traffic grooming can be implemented. This is a spectrum management technique that aggregates the traffic from multiple low-rate connections from the upper layer into high-rate optical channels. By allowing several independent traffic streams to share the bandwidth of a lightpath, it is possible to maximize the amount of traffic on each lightpath, thus minimizing the number of lightpaths [17]. Moreover, if multiple demands are groomed in the same optical tunnel, better transponder utilization is obtained. There are also substantial spectrum savings when several low-capacity demands having the same source and destination nodes are not separated by guardbands, as they are only needed between different optical tunnels [18]. By taking advantage of the appropriate traffic aggregation, grooming can provide multiple benefits to optical networking, especially in combination with other spectrum management techniques, such as proactive or reactive fragmentation minimization techniques, leading to both spectrum and transponder savings.

To the best of our knowledge, fragmentation-aware traffic grooming has not been considered for dynamic traffic in the context of SS-FONs with lane change ability. To fill this gap, we proposed a grooming algorithm to minimize the bandwidth blocking probability by minimizing the introduced SS-FONs' fragmentation metrics. We ran numerical studies assuming cloud-oriented SS-FONs and scenarios with different numbers of data centers and with the spatial continuity constraint being active and relaxed.

The rest of the paper is organized as follows. Section 2 discusses the related works, while Section 3 presents the network model. In Section 4, fragmentation metrics are described. Further, Section 5 introduces the traffic grooming algorithm, and Section 6 describes the experiments conducted. Finally, Section 7 concludes this work.

\section{Related Works}

Network fragmentation has been widely studied in the context of EONs (e.g., [13,19-22]); however, it is a relatively new topic in the context of SS-FONs. The methods, metrics, and algorithms proposed for EONs cannot be directly applied in SSFONs due to the introduced additional spatial dimension, especially when lane changes are possible. To the best of our knowledge, fragmentation metrics were studied in SS-FONs only in [23-29]. However, in these papers, the lane changes were not considered. Thus, in $[16,30]$, we proposed several fragmentation metrics that accounted for this. Note, lane changes were considered in various papers, e.g., [12,31,32], and in terms of the fragmentation/defragmentation are studied in [33-35]. However, in the referenced papers, the lane change ability was not involved in the design of the fragmentation metrics. As in [16,30], we showed that fragmentation metrics can be effectively applied in the fragmentation-aware algorithm, and we aimed to further study this topic in the context of traffic grooming.

The general topic of traffic grooming has been the subject of various books and surveys [36,37], and it has also been discussed in the context of multilayer networks [38,39]. Recently, it began gaining popularity in the context of EONs [18]. A number of algorithms have been proposed for solving the problem of optical grooming including static and dynamic traffic scenarios, allowing the establishment of lightpaths carrying several connections in a contiguous block of spectrum without inserting guardbands between them, therefore minimizing the spectrum usage or the number of transponders.

A distance-adaptive and fragmentation-aware optical grooming algorithm was presented in [40]. The objective was to minimize the maximum required spectrum to provision given traffic demands while considering the spectral continuity constraint and the transmission reach constraint. The proposed algorithm groups traffic demands with the same source node into a source set, which are further aggregated into lightpaths. To mitigate spectral fragmentation, for each of the connections that have not been groomed yet, a potential lightpath is considered, and the spectral gain is calculated. If the spectral gain is positive, then the connection is marked as groomed.

The authors of [41] investigated the traffic grooming issue with a sliceable transponder in elastic optical networks for the online traffic scenario. They implemented several 
traffic grooming policies with incorporated spectrum defragmentation. Each time a new connection request arrives, the edge weights of an auxiliary graph are adjusted. The proposed algorithm grooms incoming connections into defragmented lightpaths. The results showed a tradeoff among different traffic grooming policies as they accounted for different objectives, such as maximizing the spectrum efficiency, minimizing the blocking ratio, or minimizing the number of transponders used.

Traffic grooming was investigated in the context of SS-FONs only in a few papers, e.g., [42-45]. However, to the best of our knowledge, traffic grooming in SS-FONs has not been studied in conjunction with fragmentation metrics and with lane change ability.

\section{Network Model}

The network was modeled as a directed graph $G=(V, E)$, where $V$ is a set of network nodes and $E$ is a set of unidirectional network fiber links that interconnect them. Each link $e \in E$ is divided into $K$ spatial modes, where each mode is a single-core single-mode fiber in a fiber bundle. Each spatial mode provides an optical spectrum of $4 \mathrm{TH}$ in width divided into small frequency slots of $12.5 \mathrm{GHz}$, denoted as $S$. Let set $R$ denote the data centers (DCs) available in the network that are connected to some of the network nodes. For simplicity, we assumed that there was no limit in the bandwidth or number of connections between a network node and a DC; thus, connecting to the node is equivalent to connecting to the DC.

We assumed a similar transceiver model as in [46], i.e., network nodes are equipped with coherent transceivers operating at a fixed baud rate of 37.5 GBaud and are capable of transmitting optical carriers (OC) of $37.5 \mathrm{GHz}$ in width (3 slots). All transceivers support 4 modulation formats, namely BPSK, QPSK, 8-QAM, and 16-QAM. The bit rate and transmission distance supported by each transponder are presented in Table 1 . If the bit rate supported by the single transceiver exceeds the requested bit rate, one or more adjacent transceivers are applied to create a super-channel (SCh). Adjacent SChs were separated using guardbands of $12.5 \mathrm{GHz}$ in width.

As in EONs, we considered the spatial continuity and contiguity constraints. The former one states that in the case of a lack of spectrum converters, a super-channel has to be created using slices with the same indices along the routing path. The latter one implies that the assigned slots for the SCh should be adjacent. Depending on the scenario, the spatial continuity constraint (SCC) is considered or not. In more detail, if lane changes are assumed, the SCC is relaxed, and any input port of the network node can be freely switched to any output port. On the contrary, without lane changes, the SCC is active, and each lightpath has to be created over spatial modes with the same indices along the routing path.

Table 1. Transmission distance and supported bit rate by a single transceiver [11].

\begin{tabular}{ccc}
\hline Modulation Format & Bit-Rate (Gbps) & Transmission Range (km) \\
\hline BPSK & 50 & 6300 \\
QPSK & 100 & 3500 \\
8-QAM & 150 & 1200 \\
16-QAM & 200 & 600 \\
\hline
\end{tabular}

We considered the network traffic model proposed in [47], which considers the network operation within a given time perspective $T$ (specified by the number of time points/iterations) and ensures that the average traffic volume at each time point is equal to $B_{a v g}$ Gbps. Traffic grooming is applied on the level of a pair of communicating nodes, and at each time point $t \in T$, the model allows assessing the total bit rate $f(t, i, j)$ currently observed between each pair of nodes $(i, j): i, j \in V, i \neq j$. In order to verify whether a request has just arrived or expired, it is necessary to compare the current flow value $f(t, i, j)$ with the observation from the previous time point $f(t-1, i, j)$. Therefore, the total 
bit rate between each pair of network nodes at a given time point $t$ can be expressed as a $\operatorname{matrix} F_{t}^{V \times V}$ as in (1).

$$
F_{t}^{V \times V}=\left[\begin{array}{cccc}
0 & f(t, 0,1) & \ldots & f(t, 0,|V|) \\
f(t, 1,0) & 0 & \ldots & f(t, 1,|V|) \\
\vdots & & \ddots & \\
f(t,|V|, 0) & f(t,|V|, 1) & & f(t,|V|,|V|)
\end{array}\right]
$$

\section{Fragmentation Metrics}

As in [16], we adapted various EON link fragmentation metrics to SS-FONs by accounting for the spatial dimension. The following metrics were considered, namely external fragmentation (EF), the Shannon entropy (SE), the access blocking probability $(A B P)$ [13-15], the root of sum of squares (RSS), and the root mean-squared factor (RMSF) [48]. The set of all available metrics is denoted as $M$.

The general idea to calculate fiber $F_{\text {link }}^{m}(e)$ fragmentation using metric $m \in M$ is to average the fragmentation metrics $F_{s m}^{m}(e, k)$ calculated for each spatial mode $k \in K$ separately.

$$
F_{\text {link }}^{m}(e)=\frac{\sum_{k \in K} F_{s m}^{m}(e, k)}{|K|}
$$

Before defining particular spatial mode metrics, let us provide general considerations regarding the metrics' construction. Each spatial mode was divided into a set of free and occupied segments, where a free segment denotes a set of contiguous free slots in the fiber, while an occupied segment denotes the set of contiguous allocated ones. Intuitively, when the number of free segments increases, the number of occupied ones increases as well, and the spectrum becomes more fragmented. Therefore, the fragmentation metric should reflect these changes accordingly. When the size of free segments decreases, the fragmentation should increase as it is harder to fit a new request in smaller available spaces. Finally, when the index of last occupied slot decreases, the metric should decrease. This is a common objective function in a static problem scenario. The minimization of the highest allocated slot index usually allows allocating a higher number of requests. Next, each of the metrics is discussed.

External fragmentation (EF) originates from computer memory management, where it is defined as the ratio between the largest free memory block and the sum of all available blocks. Analogously, for network fragmentation, it is defined as the ratio of the size of the largest free segment over the sum of the sizes of all free segments (Equation (3)). $\Gamma(e, k)$ denotes the set of free segments on spatial mode $k \in K$ of link $e \in E$, while $\left|\gamma_{e k}\right|$ is the size of the segment $\gamma_{e k} \in \Gamma(e, k)$ (number of slots). The metric increases when the size of the free segment decreases; however, sometimes, it may not be meaningful, as it does not consider the sizes of the segments other than the largest one.

$$
F_{\text {link }}^{E F}(e)=1-\frac{1}{|K|} \cdot \sum_{k \in K} \frac{\max _{\gamma_{e k} \in \Gamma(e, k)}\left|\gamma_{e k}\right|}{\sum_{\gamma_{e k} \in \Gamma(e, k)}\left|\gamma_{e k}\right|}
$$

The Shannon entropy (SE) was originally applied in information theory to calculate the amount of information available in the message (Equation (4)). It accounts for the size of all free segments and promotes larger ones by taking the inversion of particular segment sizes in a logarithmic function. Thus, the SE overcomes the limitations of the EF, as the EF accounts only for the size of the largest free segment and the summary size of the available slots.

$$
F_{e}^{S E}=\frac{1}{|K|} \cdot \sum_{k \in K} \sum_{\gamma_{e k} \in \Gamma(e, k)} \frac{\left|\gamma_{e k}\right|}{|S|} \cdot \ln \frac{|S|}{\left|\gamma_{e k}\right|}
$$


The access blocking probability (ABP) additionally considers the granularity of the transceiver model (Equation (5)). The granularity refers to the possible sizes of allocated SChs according to the model including guardbands, denoted as $G$. Based on the assumptions, $G=\{g: g=3 n+1\}$, where $n \in \mathbb{N}^{+}$. The ABP metric evaluates how many SChs can be created simultaneously in the available free segments for each transceiver granularity and divides this number by the amount of SChs that could be assigned if all the free blocks were adjacent, thus forming one large free segment. In contrast to the previous metrics, the ABP accounts for the exact possible sizes of SChs created in the network.

$$
F_{e}^{A B P}=1-\frac{1}{|K|} \cdot \sum_{k \in K} \frac{\sum_{\gamma_{e k} \in \Gamma(e, k)} \sum_{g \in G}\left\lfloor\left|\gamma_{e k}\right| / g\right\rfloor}{\sum_{g \in G}\left\lfloor\left(\sum_{\gamma_{e k} \in \Gamma(e, k)}\left|\gamma_{e k}\right|\right) / g\right\rfloor}
$$

The root of sum of squares (RSS) promotes a network state where a small number of large free segments exists rather than a large number of smaller ones (Equation (6)). It is achieved by taking the square root of the sum of squares of particular free segment sizes, and it can be justified, as it is easier to assign a new request to larger free segments.

$$
F_{e}^{R S S}=1-\frac{1}{|K|} \cdot \sum_{k \in K} \frac{\sqrt{\sum_{\gamma_{e k} \in \Gamma(e, k)}\left(\left|\gamma_{e k}\right|^{2}\right)}}{\sum_{\gamma_{e k} \in \Gamma(e, k)}\left|\gamma_{e k}\right|}
$$

The root mean-squared factor (RMSF) extends the RSS concept by modifying how the square root of free segments impacts the metric and by accounting for the index of the highest allocated slot on the spatial mode $s_{e k}^{\max }$ (Equation (7)). In particular, the RMSF metric increases when: (i) the index of the highest allocated slot on each spatial mode increases; (ii) the number of free segments increases; (iii) the size of free segments decreases. When comparing to the RSS metric, the RMSF metric has an unbounded value range from 0 to infinity instead of 0 to 1 . If it is necessary to create an upper bound for the RMSF metric, this is possible for a given number of slots. The metric has the highest value when the segments are repeatedly free and occupied, each of size 1 . Assuming that $|S|$ slots are in the network, the upper bound contains $|S| / 2$ free segments, each of size one, and the highest allocated slot index is $|S|$. In such a case, the RMSF metric is equal to $|S|^{2} / 2$. However, it is irrelevant for most of the time as the metrics are used to quantitatively compare various network states, not to qualitatively describe the fragmentation of a particular state.

$$
F_{e}^{R M S F}=\frac{1}{|K|} \cdot \sum_{k \in K} \frac{s_{e k}^{\max } \cdot|\Gamma(e, k)|}{\sqrt{\frac{\sum_{\gamma_{e k} \in \Gamma(e, k)}\left|\gamma_{e k}\right|^{2}}{|\Gamma(e, k)|}}}
$$

Finally, network fragmentation $F_{\text {net }}^{m}$ is calculated as the average over the fragmentation of all the network's links. $F_{\text {link }}^{m}(e)$ uses the link fragmentation metric $m \in M$.

$$
F_{\text {net }}^{m}=\frac{\sum_{e \in E} F_{\text {link }}^{m}(e)}{|E|} \cdot \frac{s_{\text {max }}}{|S|},
$$

In Equation (8), network fragmentation is proportional to the index of the highest allocated slice $s_{\max }$ in the network. The minimization of the highest allocated slice index results in leaving the remaining upper parts of the spectrum available for new requests, and based on various experiments, these tend to accommodate more requests. Thus, the network fragmentation should account for this fact.

\section{Optimization Algorithm}

In this section, the traffic grooming, routing, and spectrum assignment (TG-RSSA) algorithm is proposed. In more detail, it grooms and releases traffic in available optical channels. If the available allocated resources are not sufficient to accommodate the increasing traffic, new optical channels are established. The decision on how these issues are 
addressed can significantly affect the network performance. To this end, we considered several grooming strategies $\gamma$ and allocation algorithms $\lambda$. The first one determines how the traffic is groomed and released from the already allocated channels, while the latter one defines which SChs should be used to create new optical corridors when the need for a new one emerges. Moreover, we assumed that the empty SChs (i.e., the ones that do not carry any traffic) should be removed from the network immediately to allow other requests to have these resources assigned.

\subsection{Grooming Strategies}

Due to the changes of the overall traffic volume between network nodes, it is required to adjust the allocated bandwidth in established SChs in the optical fibers accordingly. In particular, if the traffic increases between two nodes and there are some free (unoccupied) resources in the SChs connecting those nodes, one may decide to use them to deliver the traffic. Analogously, if the traffic decreases, the resources can be freed in those SChs. In this section, various grooming strategies $\gamma$ are described that define how requests are aggregated in the optical corridors. In more detail, if more than one super-channel is available, it is required to decide in which one the traffic is increased (decreased) firstly:

- BT (bottom-top) - groom traffic in the available channels starting from the bottom of the spectrum, and release the traffic from the channels starting from the top of the spectrum;

- $\quad$ LFLR (largest fill, largest release)—groom traffic in the available channels starting from the ones with the largest number of optical carriers, and release the traffic from the channels starting from the ones with the largest number of optical carriers;

- LFSR (largest fill, smallest release)—groom traffic in the available channels starting from the ones with the largest number of optical carriers, and release the traffic from the channels starting from the ones with the smallest number of optical carriers;

- SFLR (smallest fill, largest release) — groom traffic in the available channels starting from the ones with the smallest number of optical carriers, and release the traffic from the channels starting from the ones with the largest number of optical carriers;

- $\quad$ SFSR (smallest fill, smallest release) - groom traffic in the available channels starting from the ones with the smallest number of optical carriers, and release the traffic from the channels starting from the ones with the smallest number of optical carriers.

\subsection{Allocation Algorithm}

When the available capacity in the allocated SChs is exhausted, it is required to create a new SCh to mitigate the increasing traffic requirements. In this work, we applied three allocation algorithms $\lambda$, namely the first fit (FF), k-first fit (kFF), and fragmentationaware (FragA) algorithms [30], using the presented fragmentation metrics. The proposed algorithms are responsible for finding a SCh of the requested size (in terms of subcarriers) between given network nodes, addressing continuity and contiguity constraints and, depending on the scenario, the spatial continuity constraint:

- $\quad$ First fit (FF) - This is a well-known method that computes (or uses a pre-computed) shortest path between given nodes and finds a SCh with the lowest starting slot index. Note, as there are multiple spatial modes, the SCh with the lowest starting slot index may not be the first one found;

- $\quad$-first fit ( $\mathrm{kFF}$ - - Similar to FF, it looks for a SCh with the lowest starting slot index. However, it considers the k-shortest paths. If a SCh is not found on a certain path, it tries to create one on the consecutive (longer) ones;

- Fragmentation-aware (FragA) algorithm [30]-The FragA algorithm aims to minimize the network fragmentation by selecting a SCh that minimizes the fragmentation. The fragmentation is calculated according to the metric passed as a parameter. In more detail, for a given request, it finds a SCh with the lowest starting slot index on each k-shortest path. Next, the SChs are compared in terms of how they will affect the 
network fragmentation, and the one that will decrease it the most (or increase it the least) is selected.

\subsection{Traffic Grooming Routing and Spectrum Assignment Algorithm}

Let us denote $[\cdot]$ as the accessing operator of an element in a vector/list, e.g., $\mathbf{a}[i]$ is the $i$-th element of vector a. Similarly, $[\cdot, \cdot]$ is an accessing operator of an element in a matrix, e.g., $\mathbf{A}[i, j]$ is the element located in the $i$-th row and $j$-th column of matrix A. Moreover, we assumed that each SCh had the following properties: (i) flow $f(s c h)$, representing the traffic groomed in the SCh; (ii) capacity $c(s c h)$, defining the maximum flow that the SCh can accommodate.

Algorithm 1 presents the pseudocode of TG-RSSA. It takes three input variables-time perspective $T$, where traffic changes, traffic grooming strategy $\gamma$, and the channel allocation algorithm $\gamma$. For each time point $t \in T$, a traffic matrix $\mathbf{F}_{t}^{V \times V}$ can be expressed as in (1). The grooming strategy determines how the traffic is groomed and released in the available channels, while the allocation algorithm is applied when the assignment of a new optical corridor is needed. In Lines 2-5, variables are initialized, where $f^{t o t, s}$ and $f^{t o t, r}$ are the summary served and rejected bandwidth and $\mathbf{F}_{0}^{V \times V}$ is the initially allocated traffic matrix that is equal to a matrix of size $V \times V$ filled with zeros. Allocated requests for each node pair are stored in $\mathbf{A}^{V \times V}$, where $\mathbf{A}^{V \times V}[u, v]$ is a vector of SChs allocated between nodes $u$ and $v$. In the algorithm, traffic matrices are processed one-by-one for each time point (Line 6). Firstly, the traffic change for each node pair $\Delta \mathbf{F}^{V} \times V$ is calculated by subtracting the current traffic from the one in the previous time point. Pairs of nodes are divided into two sets $P_{d e c}$ and $P_{i n c}$. In $P_{d e c}$, pairs are stored for which the traffic is decreasing at the current time point, while in $P_{i n c}$, for which it is increasing. Firstly, traffic is released from the optical channels. To this end, for each node pair with decreasing summary flow (Line 10), a ReleaseTrafficFromChannels procedure is called. This procedure is responsible for removing specified flow from optical channels between a given pair of nodes according to the $\gamma$ strategy (Line 11). It returns the set of SChs $S C h^{u \rightarrow v}$ that are empty (do not carry any traffic) and that are released from the network (Lines 12-14). Next, the process of increasing the traffic between pairs of network nodes begins (Line 14). Firstly, node pairs are sorted in decreasing order of traffic increase between each pair, i.e., firstly, nodes are processed for which the summary flow increased the most. As it is not possible to determine whether all traffic can be groomed in the network in advance, variable $\mathbf{F}^{V \times V} t^{\prime}$ defines the actual value of traffic realized at each time point. Initially, it is equal to the value of $\mathbf{F}_{t}^{V \times V}$, and it is decreased accordingly if some of the bandwidth is rejected. Next, for each node pair with increasing traffic, it is checked if any new optical channels are required and traffic is groomed in already or newly allocated channels (Lines 17-21). Firstly, method FindAndAllocateChannels allocates new optical channels needed to support the flow increase. Note, the currently allocated channels can be sufficient, and in such a case, any new channel is not allocated. The procedure returns flow $f_{t}^{r e j}$, which cannot be accommodated in the current and new channels between the given node pairs. Next, the traffic is groomed in the available channels (Line 20) according to the $\gamma$ strategy. If any flow is rejected for the considered node pair, it is subtracted from the actually allocated traffic $\mathbf{F}^{V \times V} t^{\prime}$. After processing each node pair, in Lines 22-23, the total summary served and rejected flows are updated based on the matrix of the actually realized traffic $\mathbf{F}^{V \times V} t^{\prime}$ and the initially requested traffic matrix $\mathbf{F}_{t}^{V \times V}$. Finally, the requested traffic matrix is updated, so the requested traffic change in Line 7 can be calculated correctly in the next iteration. The algorithm continues its execution until all of the traffic matrices are considered.

Algorithm 2 presents the ReleaseTrafficFromChannels procedure responsible for releasing groomed traffic from optical channels between node pairs $u \in V$ and $v \in V$. The amount of flow to release is defined by input parameter $f_{\text {dec }}$. In Line 2, the list of optical channels $S C h^{u \rightarrow v}$ between node pairs $u$ and $v$ is obtained from the matrix of allocated channels $\mathbf{A}^{V \times V}$. The channels are sorted according to the grooming strategy $\gamma$ (Line 3 ). The presented example assumes the BT strategy, where traffic is released from SChs with 
the higher starting slot index first. Note that changing grooming strategy $\gamma$ only affects this line of pseudocode. In Lines 4-6, variables are initialized: iteration counter $i$, set of empty SChs $S C h_{\text {empty }}$, and the remaining flow $f_{\text {rem }}$ that should be released from the channels. Next, the SChs are processed one-by-one untilall of the remaining flow $f_{\text {rem }}$ is released from them (Line 7). For each SCh, it is required either to release part of the flow (Line 9) or empty it completely, depending on the value of the remaining flow $f_{\text {rem }}$. In the first case, the remaining flow $f_{\text {rem }}$ is released from the SChs' flow. In the second case, the $\mathrm{SCh}$ becomes empty (no traffic is transmitted using this channel), and it is added to $S C h_{\text {empty. }}$. When all the requested flow has been emptied from the channels, the set of empty channels is returned from the method.

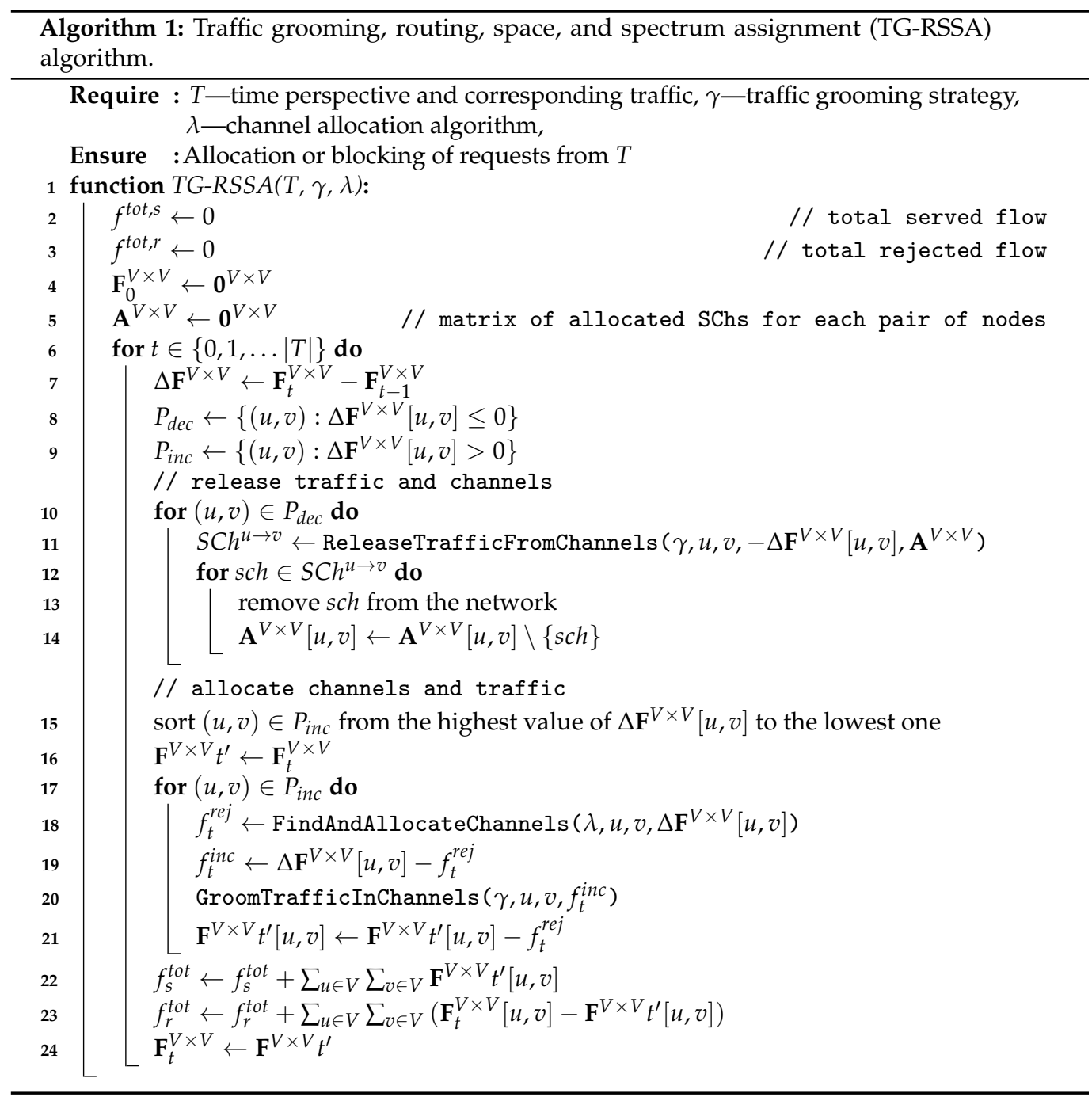

Algorithm 3 describes the GroomTrafficInChannels procedure that grooms traffic in the available channels between nodes $u \in V$ and $v \in V$. The amount of flow to groom is defined by input variable $f_{\text {add }}$. The pseudocode is similar to the one presented in Algorithm 2. Firstly, the set of SChs SCh ${ }^{u \rightarrow v}$ between nodes $u$ and $v$ is obtained and sorted according to the grooming strategy $\gamma$. The example is presented for the BT strategy; however, when changing strategies, only Line 3 is affected. The variable $f_{\text {rem }}$ holds the value of traffic that needs to be groomed in the channels, and it is initially equal to $f_{\text {add }}$. The available SChs $S C h^{u \rightarrow v}$ are processed consecutively, until not all the requested flow is groomed. In particular, if the SChs' sch residual capacity is higher than the remaining flow $f_{\text {rem }}$, the remaining flow is fully added to the current $\mathrm{SCh}$, and procedure ends its 
execution. Otherwise, the flow of the SCh is increased to its full capacity, and the remaining flow is added to the next SCh.
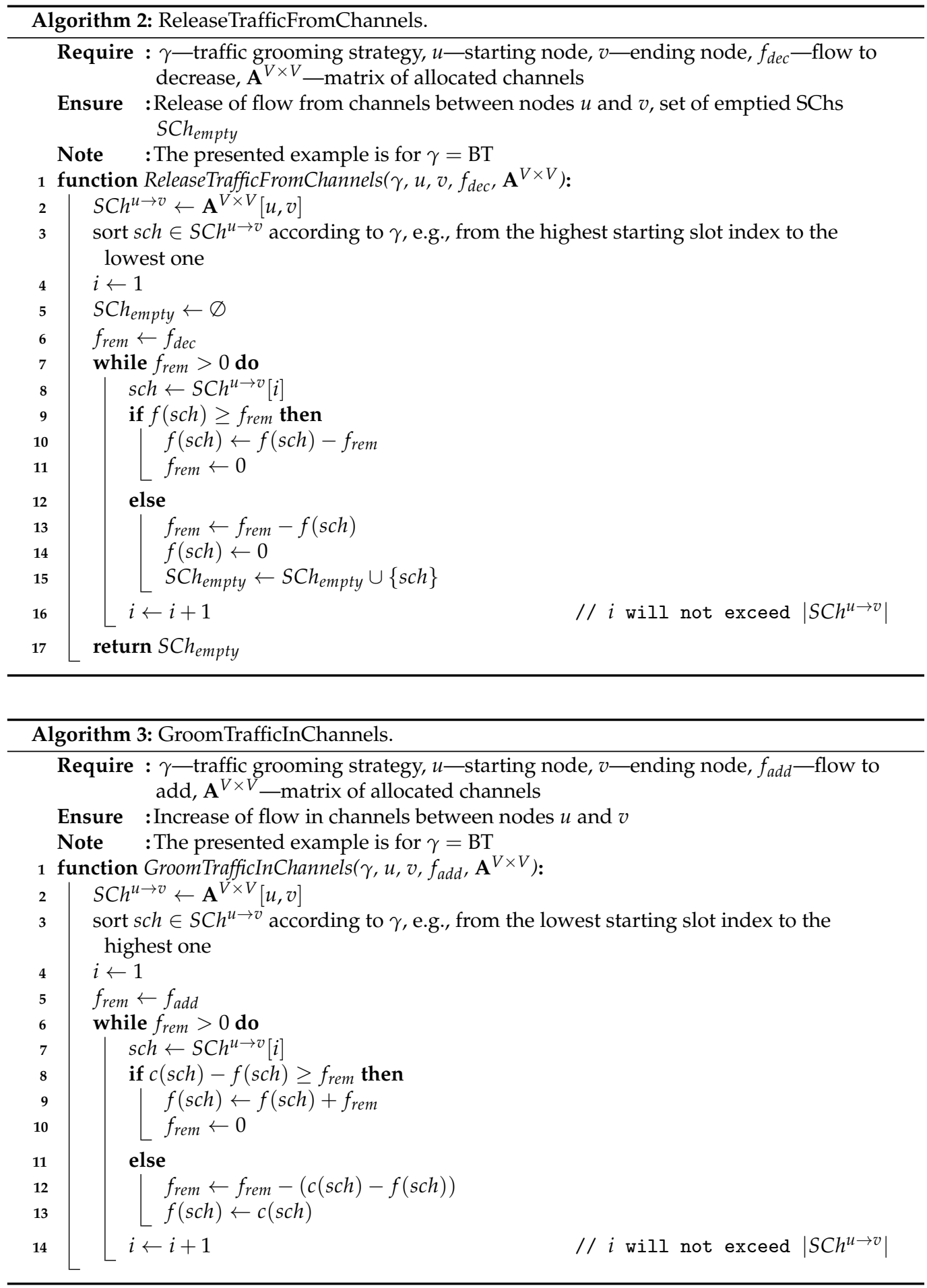


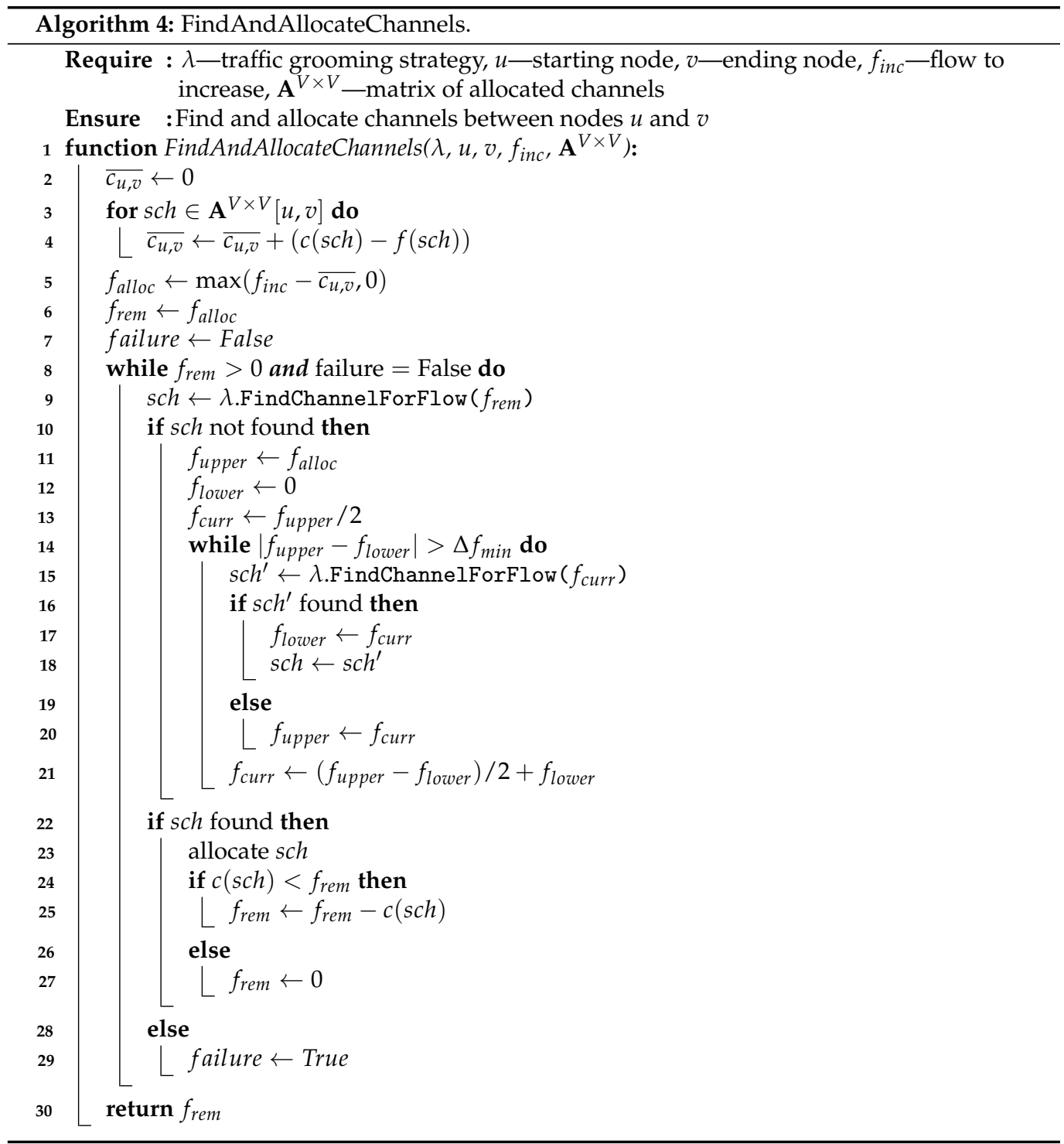

The FindAndAllocateChannels procedure is presented in Algorithm 4. It is responsible for evaluating if new optical channels are required to be allocated to support the flow increase $f_{\text {inc }}$ between nodes $u$ and $v$, or if the currently available ones are sufficient. In the former case, it allocates SChs according to the provided allocation algorithm $\lambda$. Firstly, the residual flow $\overline{\mathcal{C}_{u, v}}$ is calculated in the available SChs (Lines 2-4). In Line 5, it is calculated if the current SChs can accommodate the traffic increase and, if not, how much flow $f_{\text {alloc }}$ should be allocated to the newly created SChs. Note that there may not exist a contiguous spectrum block with capacity supporting the requested additional flow. In such a case, it is necessary to allocate multiple small SChs. Finally, when the network is congested, it may not be possible to find SChs of sufficient sizes to support all the flow. All of these cases should be considered, and to this end, the $f_{\text {rem }}$ variable is created, indicating how much residual capacity is yet required while finding SChs and the failure variable, which is set to True if it is not possible to find more SChs for a given node pair. In more detail, in the loop (Line 8), new SChs are found until either all the flow is addressed $\left(f_{\text {rem }}=0\right)$ or it is not possible to find new SChs $($ failure $=$ True $)$. Firstly, the SCh supporting all remaining flow $f_{\text {rem }}$ is sought using the FindChannelForFlow method (Line 9). The FindChannelForFlow method finds the new SCh of at least the bit rate specified as a parameter, using the allocation algorithm $\lambda$, and returns nothing if it is not possible to find such a channel. Note that the capacity of the found SCh may be a little higher than the requested one due to the transceivers' granularity and applied modulation format. If the SCh supporting 
all the remaining flow is not found (Line 10), the algorithm looks for a SCh of a smaller capacity. As it is not known in advance what the maximum supported flow of smaller available $\mathrm{SCh}$ is, the binary search is applied to find this (Lines 11-21). Let us denote $\Delta f_{\text {min }}$ as the minimum flow granularity supported by a single transceiver using the least effective modulation format (according to the network model, it is $100 \mathrm{Gbps}$ ). It is sufficient to find the supported flow in the binary search with the $\Delta f_{\min }$ accuracy (Line 14). Next, in Line 22, the algorithm checks whether any SCh is found. If so, it is allocated, and the remaining flow $f_{\text {rem }}$ is updated according to the SCh's capacity. Otherwise, the variable failure is set to True to break the search for new SChs. In the end, the algorithm returns the value of the flow $f_{\text {rem }}$ that cannot be allocated in the created SChs.

Note, depending on the selection of the allocation algorithm, we refer to the traffic grooming algorithm as first fit traffic grooming (FF-TG), k-first fit traffic grooming (kFF-TG), and fragmentation-aware traffic grooming (FragA-TG).

\section{Numerical Experiments}

The numerical experiments were conducted to evaluate the performance of the proposed fragmentation-aware traffic grooming algorithm in an SS-FON. A European network (Euro28) topology was considered with $|V|=28$ nodes, $|E|=82$ links, and $625 \mathrm{~km}$ of average link length (Figure 1). We assumed that each network link was a single-mode fiber bundle with 7 spatial modes. Each spatial mode provided $4 \mathrm{THz}$ of optical spectrum divided into flexible grid of 320 slots, each of $12.5 \mathrm{GHz}$ in width. In the network, data centers (DCs) were located at some of the network nodes. For simplicity, we assumed that the number of connections and the bandwidth between a network node and a DC were unlimited; thus, connections to the network nodes were equivalent to the connections to the DCs connected to them. Three scenarios were analyzed, where the number of data centers $|R|$ was equal to $\{3,7,11\}$. The location of the data centers is presented in Figure 2, where the colored nodes represent the ones connected to the data centers. We evaluated two cases, with the spatial continuity constraint (SCC) active and with the SCC relaxed. The former one allowed decreasing the network nodes' cost and complexity, but resulted in a higher restriction regarding the traffic allocation. The latter one allowed for higher flexibility at the expense of higher cost. Moreover, we assumed that the requests were allocated using only spectral SChs, i.e., that the SCh did not span across several spatial modes on a single fiber.

We used the network traffic model proposed in [47]. The model considers network operation within a given time perspective $T$ (specified by the number of time points/iterations) and ensures that the average traffic volume a each time point is equal to $B_{\text {avg }}$ Gbps. Traffic grooming was applied on the level of a pair of communicating nodes, and at each time point, the model allowed assessing the total bit rate currently observed between each pair of nodes. In order to verify whether a request had just arrived or expired, it was necessary to compare the current flow value with the observation from a previous time point. Four traffic types were considered-city-to-city, city-to-DC, DC-to-city, and DC-to-DC. City-to-city was observed between each pair of communicating nodes and represents a general-purpose data transmission between different cities. City-to-DC and DC-to-city affect each pair of a city and assigned DC node (each city node is assigned to the closest DC from $|R|$ available DCs) and reflect a request and provision of DC-related services. Eventually, DC-to-DC occurs between each pair of DC nodes and expresses an inter-DC synchronization. For a particular pair of communicating nodes, the time process of each traffic type was modeled using a trigonometric sine function, while the entire traffic was a sum of sine functions corresponding to the traffic types observed for that pair of nodes. The parameters of the sine functions (i.e., amplitudes, pulsations, initial phases) were properly designed based on the real characteristics of the cities corresponding to the network nodes (including gross domestic product and geographical distance). For a detailed description of the signals, refer to [47]. In this paper, we assumed $T=10,000$, and $B_{\text {avg }}$ differed depending on the number of DCs. For 3 DCs, it ranged from 675 to 850 Tbps, for 7 DCs from 575 to $750 \mathrm{Tbps}$, 
while for 11 DCs from 475 to 625 Tbps. To enable the network to reach a steady state, first, 1000 time points were not considered in the evaluation. For each pair of network nodes, the $k=3$ shortest candidate paths were computed.

To measure the network performance, the bandwidth blocking probability was used. It is defined as the ratio of the summary bit rate of blocked requests to the summary bit rate of all requests offered to the network. To increase the reliability of the results, the blocking probability was calculated every 100 iterations, which resulted in 90 samples per single test scenario. During the experiments, three traffic grooming algorithms were considered: the proposed fragmentation-aware traffic grooming (FragA-TG) algorithm, and two reference ones, FF-TG and kFF-TG.

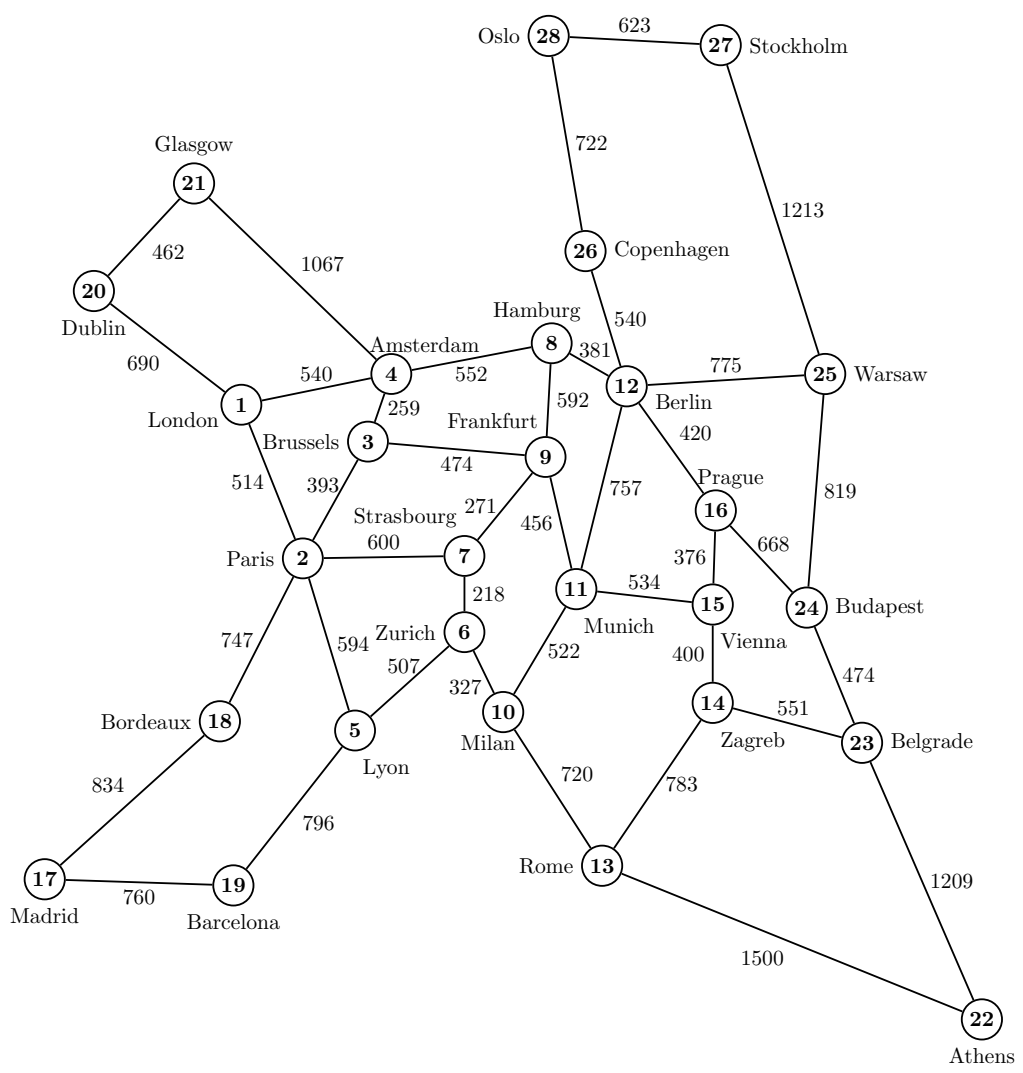

Figure 1. European network topology.

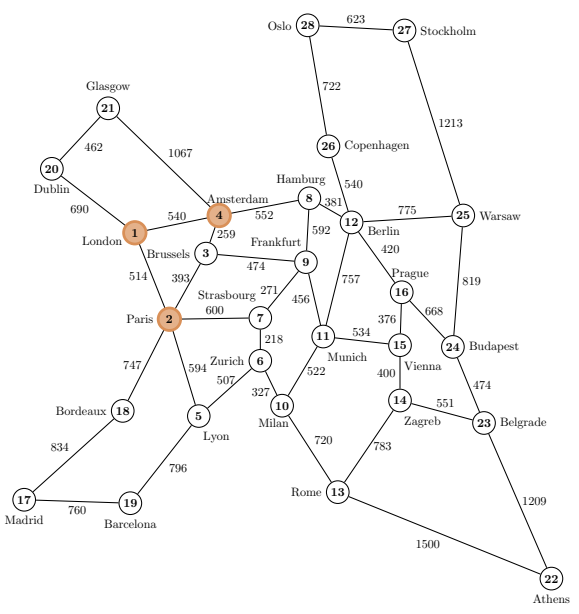

(a) 3 data centers

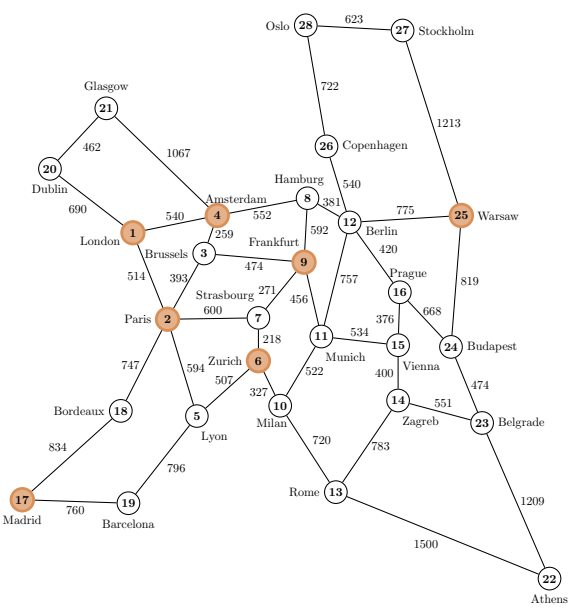

(b) 7 data centers

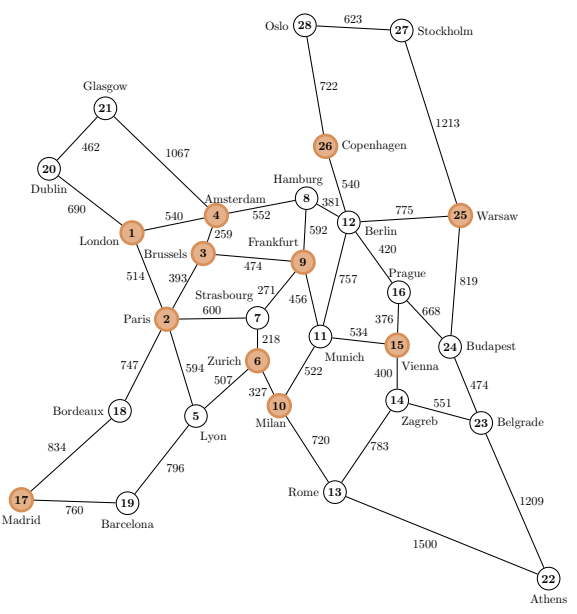

(c) 11 data centers

Figure 2. Data centers' location in the European network topology. 
In the first experiment, various traffic grooming strategies were evaluated, namely the BT, LFLR, LFSR, SFLR, and SFSR for FF-TG and kFF-TG. Figures 3 and 4 present the $B B P$ as a function of the average traffic volume between each pair of nodes for various allocation strategies, for FF-TG and kFF-TG, respectively. The results are reported for different numbers of DCs, with the SCC active and relaxed. As can be observed, for different numbers of DCs, different sets of allocation strategies decreased the BBP. In particular, for three DCs, these were LFLR and SFLR, while for seven and eleven DCs, these corresponded to BT, LFSR, and SFSR. With the increase of the DCs' number, it was more profitable to release traffic from smaller optical corridors first. Smaller SChs are usually built on top of the larger ones, which are created during the initial network setup. This was somehow confirmed by the similar good performance of the BT strategy that releases the channels from the top of the spectrum first. When the number of DCs increased, the DC-DC and client-to-DC connections dominated the network, creating complex traffic patterns that could change during the network lifetime. Therefore, releasing traffic from small requests first sped up the recycling of the optical corridors used and the allocation of the same resources for other SChs. On the contrary, when the number of DCs was small, city-to-city was the dominating traffic type. In such a case, traffic was distributed more evenly in the network, and a more promising approach was to release the traffic from the larger channels first. Such an approach increased the reusability of the already created channels without the need for the constant deallocation and allocation of the SChs on the same path. Decreasing the number of smaller SChs in favor of larger ones decreased the number of slots wasted for the guardbands. As a consequence, this allowed a lower blocking probability and allocating a higher amount of traffic. In particular, around $1 \%$ was achieved approximately for 750, 600, and 475 Tbps of average traffic volume for 3, 7, and 11 DCs, respectively.

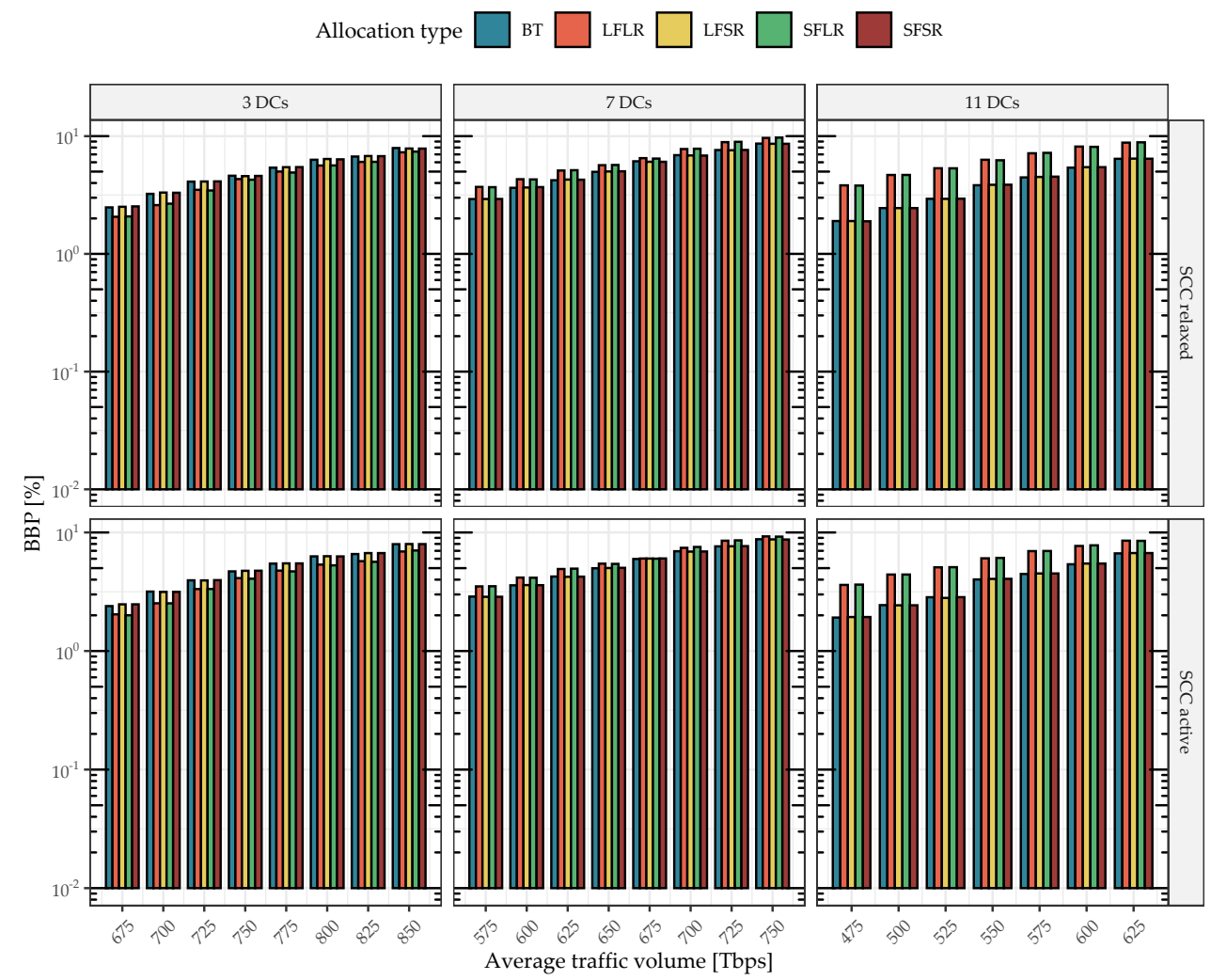

Figure 3. BBP as a function of the average traffic volume for various grooming strategies for the FF-TG algorithm. 


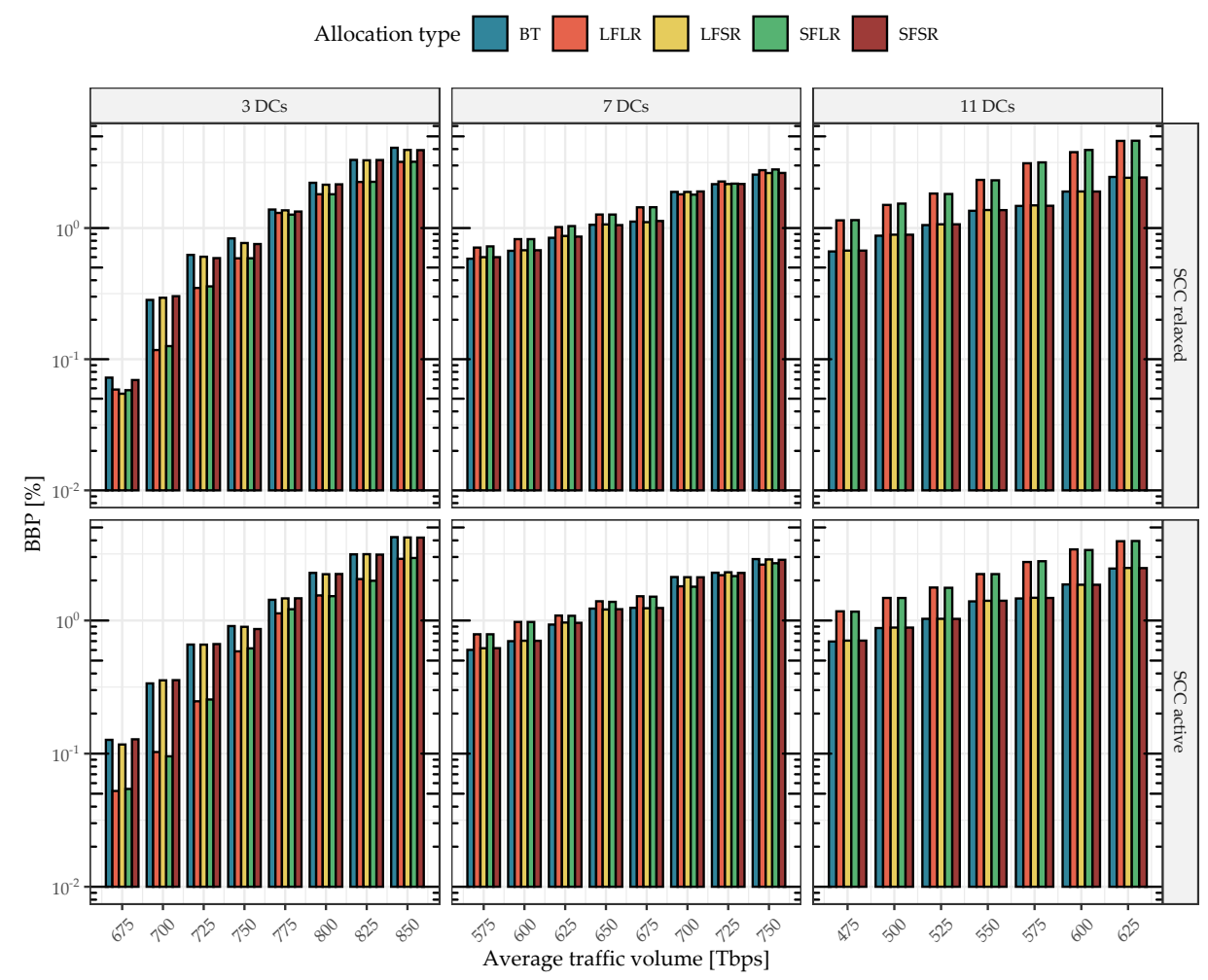

Figure 4. BBP as a function of the average traffic volume for various grooming strategies for the kFF-TG algorithm.

These results are further confirmed by Tables 2 and 3, where the average ranking and percentage difference from the minimum result are presented, respectively, for various grooming strategies. The presented results were averaged over both the FF-TG and kFF-TG algorithms. We can observe that in the case of three and eleven DCs, the difference between various grooming strategies was more significant. For further experiments, the grooming strategies that were the best (according to Table 3) were selected for each test case (number of DCs and the SCC scenario).

Next, different fragmentation metrics were evaluated in the FragA-TG algorithm, namely the EF, SE, ABP, RMSF, and RSS. For each test scenario, the best allocation type from the previous experiment was used. Figure 5 presents the BBP for various fragmentation metrics applied in the FragA-TG algorithm as a function of the average traffic volume. In most of the cases, the RMSF metric provided the lowest results. On average, it was $26.7 \%, 23.2 \%, 23.2 \%$, and $26.6 \%$ better than the EF, SE, ABP, and RSS, respectively. The RMSF metric accounted at the same time for the number and size of available contiguous spectrum slots, as well as the index of the highest allocated slot, suggesting that these parameters were suited to reflect the network fragmentation.

Table 2. Ranking of various grooming strategies for the FF-TG and kFF-TG algorithms.

\begin{tabular}{ccccccc}
\hline \multicolumn{2}{c}{ Scenario } & \multicolumn{5}{c}{ Allocation Type } \\
\hline Data Centers & SCC & BT & LFLR & LFSR & SFLR & SFSR \\
\hline 3 & relaxed & 3.368 & 2.895 & 3.360 & $\mathbf{2 . 8 9 4}$ & 3.357 \\
3 & active & 3.371 & 2.727 & 3.387 & $\mathbf{2 . 7 2 2}$ & 3.384 \\
7 & relaxed & $\mathbf{3 . 5 3 0}$ & 4.031 & 3.535 & 4.037 & 3.541 \\
7 & active & $\mathbf{3 . 6 0 1}$ & 3.902 & 3.601 & 3.910 & 3.602 \\
11 & relaxed & $\mathbf{2 . 6 8 9}$ & 4.529 & 2.702 & 4.544 & 2.703 \\
11 & active & $\mathbf{2 . 7 1 4}$ & 4.276 & 2.731 & 4.288 & 2.734 \\
\hline
\end{tabular}


Table 3. Average percentage and minimum result for various allocation strategies for the first fit and k-first fit algorithms.

\begin{tabular}{ccccccc}
\hline \multicolumn{2}{c}{ Scenario } & \multicolumn{5}{c}{ Allocation Type } \\
\hline Data Centers & SCC & BT & LFLR & LFSR & SFLR & SFSR \\
\hline 3 & relaxed & 4413.8 & $\mathbf{3 4 . 9}$ & 4771.1 & 44.9 & 5037.8 \\
3 & active & 4241.3 & 49.1 & 4596.0 & $\mathbf{1 8 . 5}$ & 4980.4 \\
7 & relaxed & $\mathbf{4 . 2}$ & 20.5 & 5.0 & 20.6 & 5.1 \\
7 & active & $\mathbf{5 . 7}$ & 17.4 & 6.1 & 17.4 & 6.1 \\
11 & relaxed & $\mathbf{0 . 7}$ & 78.6 & 1.4 & 79.5 & 1.4 \\
11 & active & $\mathbf{0 . 7}$ & 67.7 & 1.3 & 67.9 & 1.4 \\
\hline
\end{tabular}

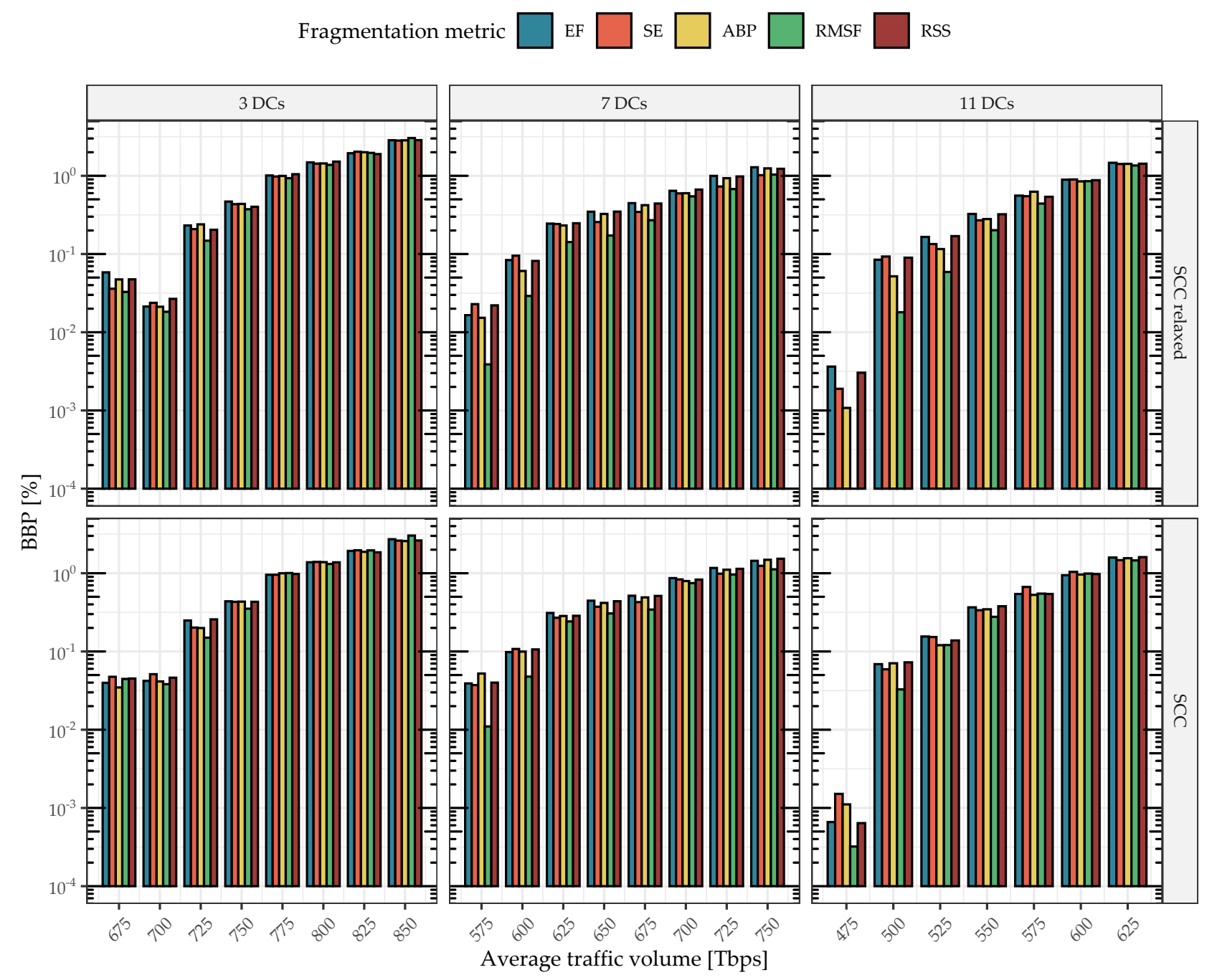

Figure 5. BBP as a function of various fragmentation metrics for the FragA-TG algorithm.

As the RMSF metric allows reducing the BBP the most, it was used in further experiments. In Figure 6, the FragA-TG algorithm is compared with the two reference ones, namely FF-TG and kFF-TG. In each case, FragA-TG was the best algorithm resulting in the lowest BBP. On average, the BBP for FragA-TG was $89.3 \%$ and $60.4 \%$ better than for FF-TG and kFF-TG, respectively. Moreover, when the number of DCs was higher, the amount of allocated traffic was lower for similar BBP levels due to the complex traffic patterns. However, FragA-TG can effectively reduce blocking, especially for lesser amounts of traffic. With the increase of the traffic load, fragmentation management became less effective. This 
can be reasoned, as the number of free contiguous blocks decreased when the network was congested, and all algorithms tended to select similar SChs.

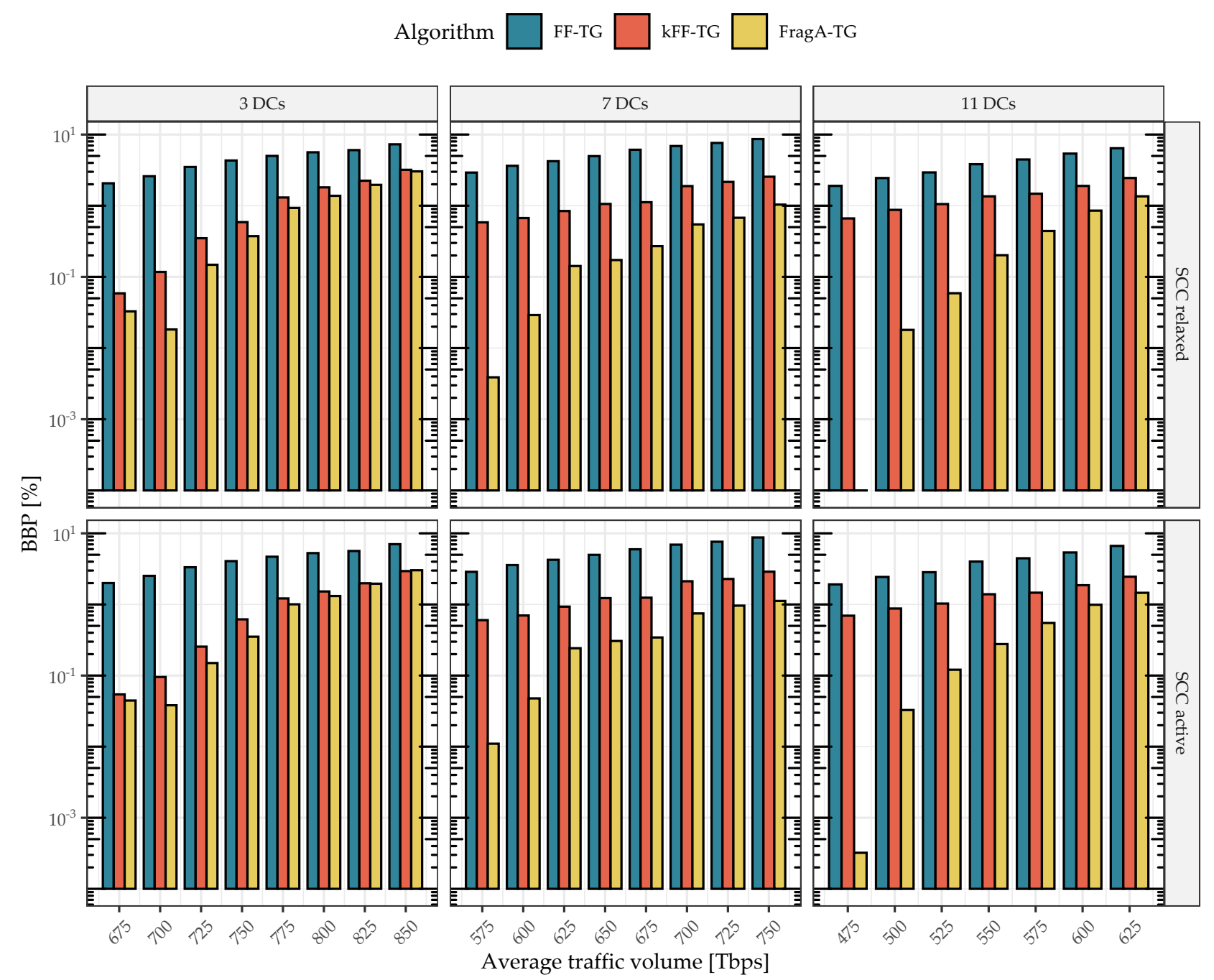

Figure 6. BBP as a function of average traffic volume for various traffic grooming algorithms.

Finally, in Figure 7, the impact of lane changes (SCC) is compared for various numbers of DCs. As can be observed, in each test case, lane changes allowed reducing network fragmentation, while the differences were the most significant for seven and eleven data centers. In particular, the average difference between the SCC relaxed and active was equal to $9.5 \%, 34.3 \%$, and $33.3 \%$ for for 3,7 , and 11 DCs. One can conclude that the spatial flexibility was more important in traffic patterns that were less uniform where certain nodes (data centers) communicated more often and certain routes were more congested. The ability to switch the traffic to another spatial mode along the route allowed reducing the blocking. On the contrary, when the traffic was more uniformly distributed, the requests could be allocated, preserving the spatial continuity more easily. 


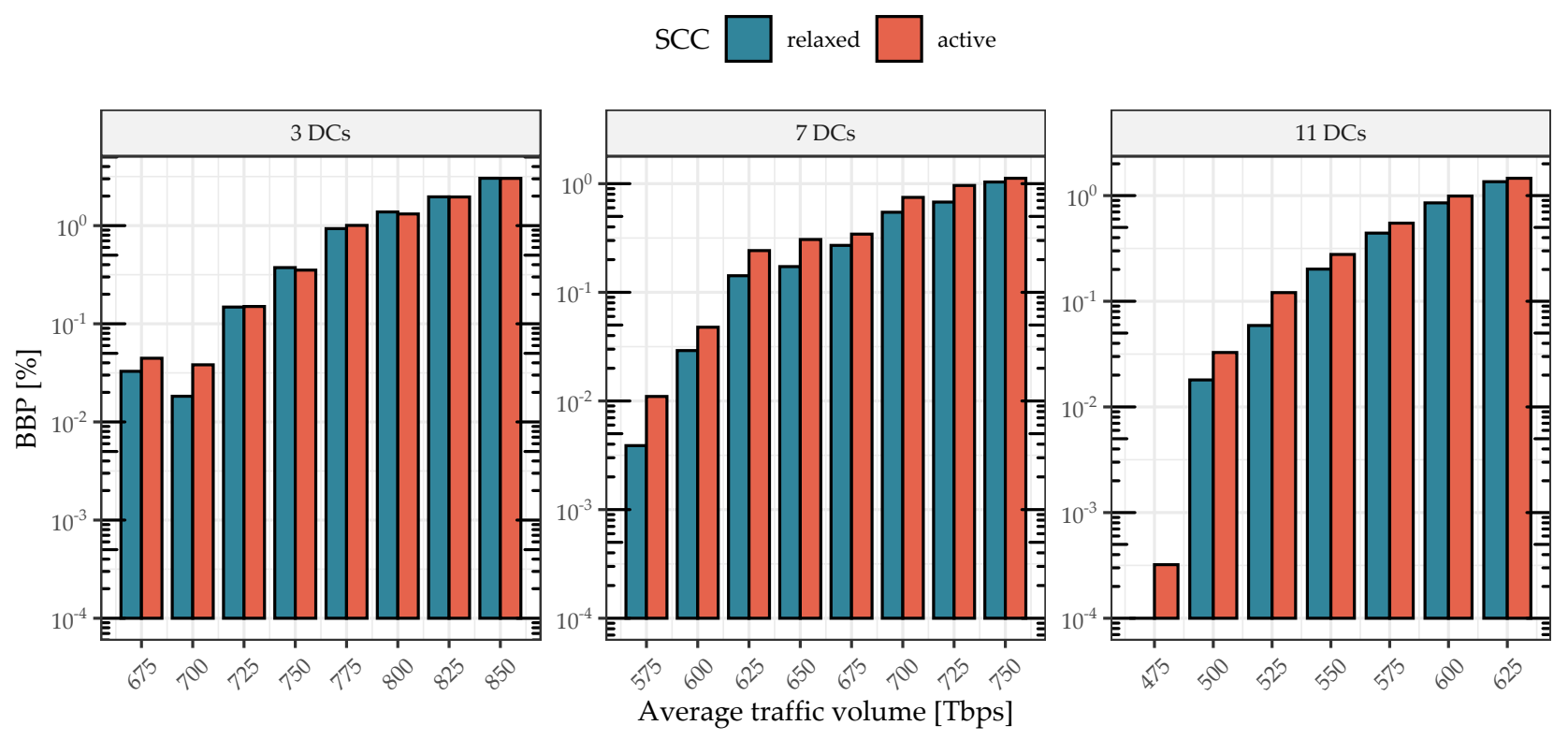

Figure 7. BBP as a function of average traffic volume for the SCC relaxed and active, for the FragA-TG algorithm.

\section{Conclusions}

In this paper, we proposed the Fragmentation-Aware Traffic Grooming (FragA-TG) algorithm in the context of SS-FONs with lane change ability for different numbers of data centers. While grooming the end-to-end traffic from pairs of network nodes, the method creates new optical corridors, minimizing the presented fragmentation metrics. According to the case study performed, this allows significantly reducing the bandwidth blocking probability (BBP), especially for smaller traffic loads and when the higher number of data centers is considered. In more detail, five different fragmentation metrics were studied, and it was shown that the root mean-squared factor metric outperformed the others. This metric accounts for both the number and size of available contiguous spectrum slots, as well as the index of the highest allocated slot, suggesting that these parameters are suited to reflect the network fragmentation. Moreover, we considered five different grooming strategies determining to and from which channels the traffic was groomed and released first. According to obtained results, the selection of the strategy should depend on the number of data centers. Furthermore, we demonstrated that, in each test case, lane changes allowed reducing network fragmentation, while the differences were the most significant for a higher number of data centers. In the future, we plan to design a machine learning model to predict the traffic and design a fragmentation-aware traffic grooming algorithm that applies those predictions to minimize the bandwidth blocking probability.

Author Contributions: Conceptualization, P.L.; methodology, P.L. and R.G.; software, P.L. and R.G.; validation, P.L., R.G., and A.K.; formal analysis, P.L. and R.G.; investigation, P.L.; resources, P.L. and R.G.; data curation, P.L. and R.G.; writing-original draft preparation, P.L., R.G., and A.K.; visualization, P.L.; supervision, P.L.; project administration, P.L.; funding acquisition, R.G. All authors read and agreed to the published version of the manuscript.

Funding: The work of P. Lechowicz and R. Goścień was supported by the National Science Centre (NCN), Poland, under Grant 2018/31/D/ST6/03041. The work of A. Knapińska was supported by the National Science Centre, Poland, under Grant 2019/35/B/ST7/04272.

Institutional Review Board Statement: Not applicable.

Informed Consent Statement: Not applicable. 
Conflicts of Interest: The authors declare no conflict of interest. The funders had no role in the design of the study; in the collection, analyses, or interpretation of data; in the writing of the manuscript; nor in the decision to publish the results.

\section{References}

1. CISCO. Cisco Visual Networking Index: Forecast and Trends, 2017-2022; Technical Report; CISCO White Paper; Cisco: San Jose, CA, USA, 2018

2. Essiambre, R.; Kramer, G.; Winzer, P.J.; Foschini, G.J.; Goebel, B. Capacity Limits of Optical Fiber Networks. J. Light. Technol. 2010, 28, 662-701. [CrossRef]

3. Winzer, P.J. Spatial Multiplexing in Fiber Optics: The 10X Scaling of Metro/Core Capacities. Bell Labs Tech. J. 2014, 19, 22-30. [CrossRef]

4. Klonidis, D.; Cugini, F.; Gerstel, O.; Jinno, M.; Lopez, V.; Palkopoulou, E.; Sekiya, M.; Siracusa, D.; Thouénon, G.; Betoule, C. Spectrally and spatially flexible optical network planning and operations. IEEE Commun. Mag. 2015, 53, 69-78. [CrossRef]

5. Klinkowski, M.; Lechowicz, P.; Walkowiak, K. Survey of resource allocation schemes and algorithms in spectrally-spatially flexible optical networking. Opt. Switch. Netw. 2018, 27, 58-78. [CrossRef]

6. Saridis, G.M.; Alexandropoulos, D.; Zervas, G.; Simeonidou, D. Survey and Evaluation of Space Division Multiplexing: From Technologies to Optical Networks. IEEE Commun. Surv. Tutor. 2015, 17, 2136-2156. [CrossRef]

7. Jinno, M.; Takara, H.; Kozicki, B.; Tsukishima, Y.; Sone, Y.; Matsuoka, S. Spectrum-efficient and scalable elastic optical path network: Architecture, benefits, and enabling technologies. IEEE Commun. Mag. 2009, 47, 66-73. [CrossRef]

8. Gerstel, O.; Jinno, M.; Lord, A.; Yoo, S.J.B. Elastic optical networking: A new dawn for the optical layer? IEEE Commun. Mag. 2012, 50, 12-20. [CrossRef]

9. Moreno-Muro, F.J.; Rumipamba-Zambrano, R.; Pavón-Marino, P.; Perelló, J.; Gené, J.M.; Spadaro, S. Evaluation of CoreContinuity-Constrained ROADMs for Flex-Grid/MCF Optical Networks. IEEE/OSA J. Opt. Commun. Netw. 2017, 9, 1041-1050. [CrossRef]

10. Marom, D.M.; Colbourne, P.D.; D’errico, A.; Fontaine, N.K.; Ikuma, Y.; Proietti, R.; Zong, L.; Rivas-Moscoso, J.M.; Tomkos, I. Survey of photonic switching architectures and technologies in support of spatially and spectrally flexible optical networking [invited]. IEEE/OSA J. Opt. Commun. Netw. 2017, 9, 1-26. [CrossRef]

11. Khodashenas, P.S.; Rivas-Moscoso, J.M.; Siracusa, D.; Pederzolli, F.; Shariati, B.; Klonidis, D.; Salvadori, E.; Tomkos, I. Comparison of Spectral and Spatial Super-Channel Allocation Schemes for SDM Networks. J. Light. Technol. 2016, 34, 2710-2716. [CrossRef]

12. Walkowiak, K.; Lechowicz, P.; Klinkowski, M.; Sen, A. ILP modeling of flexgrid SDM optical networks. In Proceedings of the 2016 17th International Telecommunications Network Strategy and Planning Symposium (Networks), Montreal, QC, Canada, 26-28 September 2016; pp. 121-126. [CrossRef]

13. Chatterjee, B.C.; Ba, S.; Oki, E. Fragmentation Problems and Management Approaches in Elastic Optical Networks: A Survey. IEEE Commun. Surv. Tutor. 2018, 20, 183-210. [CrossRef]

14. Amar, D.; Le Rouzic, E.; Brochier, N.; Auge, J.L.; Lepers, C.; Perrot, N. Spectrum fragmentation issue in flexible optical networks: Analysis and good practices. Photonic Netw. Commun. 2015, 29, 230-243. [CrossRef]

15. Wright, P.; Parker, M.C.; Lord, A. Minimum- and maximum-entropy routing and spectrum assignment for flexgrid elastic optical networking [invited]. IEEE/OSA J. Opt. Commun. Netw. 2015, 7, A66-A72. [CrossRef]

16. Lechowicz, P.; Tornatore, M.; Włodarczyk, A.; Walkowiak, K. Fragmentation metrics and fragmentation-aware algorithm for spectrally/spatially flexible optical networks. IEEE/OSA J. Opt. Commun. Netw. 2020, 12, 133-145. [CrossRef]

17. Hou, W.; Guo, L.; Wei, X. Robust and Integrated Grooming for Power- and Port-Cost-Efficient Design in IP Over WDM Networks. J. Light. Technol. 2011, 29, 3035-3047. [CrossRef]

18. Miladić-Tešić, S.; Marković, G.; Radojičić, V. Traffic grooming technique for elastic optical networks: A survey. Optik 2019, 176, 464-475. [CrossRef]

19. Chen, X.; Li, J.; Zhu, P.; Tang, R.; Chen, Z.; He, Y. Fragmentation-aware routing and spectrum allocation scheme based on distribution of traffic bandwidth in elastic optical networks. IEEE/OSA J. Opt. Commun. Netw. 2015, 7, 1064-1074. [CrossRef]

20. Pederzolli, F.; Siracusa, D.; Zanardi, A.; Galimberti, G.; Fauci, D.L.; Martinelli, G. Path-based fragmentation metric and RSA algorithms for elastic optical networks. IEEE/OSA J. Opt. Commun. Netw. 2019, 11, 15-25. [CrossRef]

21. Oki, E.; Sato, T.; Chatterjee, B.C. Spectrum Fragmentation Management in Elastic Optical Networks. In Proceedings of the 2019 21st International Conference on Transparent Optical Networks (ICTON), Angers, France, 9-13 July 2019; pp. 1-4. [CrossRef]

22. Zhu, M.; Zhang, S.; Sun, Q.; Li, G.; Chen, B.; Gu, J. Fragmentation-aware VONE in elastic optical networks. IEEE/OSA J. Opt. Commun. Netw. 2018, 10, 809-822. [CrossRef]

23. Yousefi, F.; Ghaffarpour Rahbar, A.; Ghadesi, A. Fragmentation and time aware algorithms in spectrum and spatial assignment for space division multiplexed elastic optical networks (SDM-EON). Comput. Netw. 2020, 174, 107232. [CrossRef]

24. Yousefi, F.; Rahbar, A.G. Novel fragmentation-aware algorithms for multipath routing and spectrum assignment in elastic optical networks-space division multiplexing (EON-SDM). Opt. Fiber Technol. 2018, 46, 287-296. [CrossRef]

25. Jafari-Beyrami, M.; Ghaffarpour Rahbar, A.; Hosseini, S. On-demand fragmentation-aware spectrum allocation in space division multiplexed elastic optical networks with minimized crosstalk and multipath routing. Comput. Netw. 2020, 181, 107531. [CrossRef] 
26. Trindade, S.; da Fonseca, N.L.S. Proactive Fragmentation-Aware Routing, Modulation Format, Core, and Spectrum Allocation in EON-SDM. In Proceedings of the ICC 2019-2019 IEEE International Conference Communications (ICC), Shanghai, China, 20-24 May 2019; pp. 1-6. [CrossRef]

27. Trindade, S.; da Fonseca, N.L.S. Machine Learning for Spectrum Defragmentation in Space-Division Multiplexing Elastic Optical Networks. IEEE Netw. 2020, 35, 326-332. [CrossRef]

28. Zhang, J.; Bao, B.; Yao, Q.; Ren, D.; Hu, J.; Zhao, J. 3D Fragmentation Metric and RCSA Scheme for Space Division Multiplexing Elastic Optical Networks. IEEE Access 2020, 8, 201595-201605 [CrossRef]

29. Liu, L.; Zhu, Z.; Yoo, S.J.B. 3D elastic optical networks in temporal, spectral, and spatial domains with fragmentation-aware RSSMA algorithms. In Proceedings of the 2014 European Conference on Optical Communication (ECOC), Cannes, France, 21-25 September 2014; pp. 1-3. [CrossRef]

30. Lechowicz, P.; Tornatore, M.; Włodarczyk, A.; Walkowiak, K. Fragmentation Metrics in Spectrally-Spatially Flexible Optical Networks. In Optical Network Design and Modeling; Lecture Notes in Computer Science; Springer: Berlin/Heidelberg, Germany, 2020; Volume 11616, pp. 235-247.

31. Pederzolli, F.; Siracusa, D.; Rivas-Moscoso, J.M.; Shariati, B.; Salvadori, E.; Tomkos, I. Spatial group sharing for SDM optical networks with Joint Switching. In Proceedings of the 2016 International Conference on Optical Network Design and Modeling (ONDM), Cartagena, Spain, 9-12 May 2016; pp. 1-6. [CrossRef]

32. Pederzolli, F.; Siracusa, D.; Shariati, B.; Rivas-Moscoso, J.M.; Salvadori, E.; Tomkos, I. Improving performance of spatially joint- switched space division multiplexing optical networks via spatial group sharing. IEEE/OSA J. Opt. Commun. Netw. 2017, 9, B1-B11. [CrossRef]

33. Tode, H.; Hirota, Y. Routing, Spectrum, and core and/or mode assignment on space-division multiplexing optical networks [invited]. IEEE/OSA J. Opt. Commun. Netw. 2017, 9, A99-A113. [CrossRef]

34. Meloni, G.; Fresi, F.; Imran, M.; Paolucci, F.; Cugini, F.; D’Errico, A.; Giorgi, L.; Sasaki, T.; Castoldi, P.; Pot, L. Software-Defined Defragmentation in Space-Division Multiplexing With Quasi-Hitless Fast Core Switching. J. Light. Technol. 2016, 34, 1956-1962. [CrossRef]

35. Zhao, Y.; Hu, L.; Zhu, R.; Yu, X.; Wang, X.; Zhang, J. Crosstalk-Aware Spectrum Defragmentation Based on Spectrum Compactness in Space Division Multiplexing Enabled Elastic Optical Networks With Multicore Fiber. IEEE Access 2018, 6, 15346-15355. [CrossRef]

36. Zhu, K.; Mukherjee, B. A review of traffic grooming in WDM optical networks: Architectures and challenges. Opt. Netw. Mag. 2003, 4, 55-64.

37. Dutta, R.; Kamal, A.E.; Rouskas, G.N. Traffic Grooming for Optical Networks: Foundations, Techniques and Frontiers; Springer Science \& Business Media: Berlin, Germany, 2008.

38. Zhang, S.; Tornatore, M.; Shen, G.; Zhang, J.; Mukherjee, B. Evolving traffic grooming in multi-layer flexible-grid optical networks with software-defined elasticity. J. Light. Technol. 2014, 32, 2905-2914. [CrossRef]

39. Rožić, Ć.; Klonidis, D.; Tomkos, I. A survey of multi-layer network optimization. In Proceedings of the 2016 International Conference on Optical Network Design and Modeling (ONDM), Cartagena, Spain, 9-12 May 2016; pp. 1-6.

40. Ye, Z.; Patel, A.N.; Ji, P.N.; Qiao, C. Distance-adaptive and fragmentation-aware optical traffic grooming in flexible grid optical networks. In Proceedings of the 2014 OptoElectronics and Communication Conference and Australian Conference on Optical Fibre Technology, Melbourne, VIC, Australia, 6-10 July 2014; pp. 355-356.

41. Yu, X.; Zhao, Y.; Zhang, J.; Wang, J.; Zhang, G.; Chen, X.; Zhang, J. Dynamic traffic grooming with Spectrum Engineering (TG-SE) in flexible grid optical networks. Opt. Fiber Technol. 2015, 26, 150-156. [CrossRef]

42. Oliveira, H.M.N.S.; da Fonseca, N.L.S. Protection, Routing, Spectrum and Core Allocation in EONs-SDM for Efficient Spectrum Utilization. In Proceedings of the ICC 2019-2019 IEEE International Conference on Communications (ICC), Shanghai, China, 20-24 May 2019; pp. 1-6. [CrossRef]

43. Rumipamba-Zambrano, R.; Perelló, J.; Spadaro, S. Dynamic Traffic Grooming in Joint Switching (JoS)-Enabled Flex-Grid/SDM Optical Core Networks. In Proceedings of the 2018 European Conference on Optical Communication (ECOC), Rome, Italy, 23-27 September 2018; pp. 1-3. [CrossRef]

44. Oliveira, H.M.N.S.; da Fonseca, N.L.S. Spectrum Overlap and Traffic Grooming in P-Cycle Algorithm Protected SDM Optical Networks. In Proceedings of the 2018 IEEE International Conference on Communications (ICC), Kansas City, MO, USA, 20-24 May 2018; pp. 1-6. [CrossRef]

45. Tian, R.; Zhao, Y.; Zhang, J.; Yu, X.; Li, Y.; Yu, C.; Zhang, J.; Liu, C.; Zhang, G. Dynamic traffic grooming based on auxiliary graph in spatial division multiplexing enabled elastic optical networks. In Proceedings of the 2016 15th International Conference on Optical Communications and Networks (ICOCN), Hangzhou, China, 24-27 September 2016; pp. 1-3. [CrossRef]

46. Rottondi, C.; Tornatore, M.; Gavioli, G. Optical ring metro networks with flexible Grid and distance-adaptive optical coherent transceivers. Bell Labs Tech. J. 2013, 18, 95-110. [CrossRef]

47. Goścień, R. Traffic-Aware Service Relocation in Cloud-Oriented Elastic Optical Networks. arXiv 2021, arXiv:cs.NI/2105.07653.

48. Ye, Z.; Patel, A.N.; Ji, P.N.; Qiao, C. Root mean square (RMS) factor for assessing spectral fragmentation in flexible grid optical networks. In Proceedings of the 2014 OptoElectronics and Communication Conference and Australian Conference on Optical Fibre Technology, Melbourne, VIC, Australia, 6-10 July 2014; pp. 357-358. 